\title{
FLUCTUATIONS LOCATED AT CURRENT FILAMENT BOUNDARIES IN n-GaAs
}

\author{
A. Brandl, M. Völcker* and W. Prettl \\ Institut für Angewandte Physik, Universität, \\ D-8400 Regensburg, Federal Republic of Germany \\ (Received 23 June 1989 by M. Cardona)
}

\begin{abstract}
The location of fluctuations occurring in a filamentary current flow is detected by spatially resolved photocurrent measurements using optical interband excitation in $\mathrm{n}$-GaAs at low temperatures. Current fluctuations are only observed in the filament boundaries. In the presence of an external magnetic field, characteristics like the frequency and amplitude of the spontaneous current oscillations are different for opposite edges of the filament pattern. The observed phenomena are discussed in terms of electron generation-recombination kinetics.
\end{abstract}

At low temperatures impact ionization of shallow impurities in high-purity semiconductors causes highly nonlinear current-voltage characteristics. Steady state properties of the transition from the low-conducting to the high-conducting state have been described in the framework of nonequilibrium phase transformations [1-3]. In the course of the transition to the high-conducting regime current fluctuations were observed in various semiconductor materials [4-10], which have been analyzed in terms of nonlinear dynanics and of chaos theory. Regimes of periodic, quasiperiodic, mode-locked and chaotic current fluctuations were found and the diverse scenarios like Ruelle-Takens-Newhouse, Feigenbaum and intermittency for the transition to chaotic dynamics were identified. For the occurrence of multifrequency oscillations and chaos a weak external magnetic field is necessary which represents a very sensitive control parameter for the spontaneous fluctuations [8].

The reconstruction of the spatial structure of the current flow through the material reveals a filamentary pattern in the post-breakdown regime [11-15]. Applying a low-temperature scanning electron microscope Mayer et al. have shown that current oscillations may be locked to periodic excitations of a filament boundary, leading to the conclusion that autonomous oscillations occur in the boundary [14]. In a recent investigation it was demonstrated that local optical interband excitations may be used as well to analyze filamentary current flow [15]. Compared to electron beam techniques this method has the advantage that the sample may be subjected to magnetic fields of any strength and in addition no external electrons are injected into the material. In n-GaAs epitaxial layers large asymmetries of the spatially resolved

- Permanent addreas: Max-Planck-Institut für Quantenoptik, D-8046 Garching, Federal Republic of Germany photoresponse, which are caused by an external magnetic field, have been detected [15]. In the present investigations we show that a stable current filament, formed in $\mathrm{n}$-GaAs by impact ionization of shallow donors, is destabilized by interband transitions due to laser irradiation focused on the filament boundaries. The observed current fluctuations, induced by steady state local optical excitations, are self-sustained and exhibit, in the sense of nonlinear dynamics, similar temporal behaviour as those occurring in the autonomous case. As a result, we find that in a magnetic field distinct modes of current oscillations, differing in frequency and amplitude, are located in opposite edges of the current filament.

The measurements have been carried out on an nGaAs epitaxial layer mounted in the center of a superconducting solenoid, which was immersed in liquid helium at a temperature of $1.7 \mathrm{~K}$. The magnetic field was perpendicular to the current flow and to the sample surface being parallel to the [100] crystallographic orientation. The donor concentration was $N_{D}=12 \times 10^{14} \mathrm{~cm}^{-3}$ at a compensation ratio of $92 \%$. The mobility of the electrons was $\mu=4.5 \times 10^{4} \mathrm{~cm}^{2} / \mathrm{Vs}_{\mathrm{s}}$ at $77 \mathrm{~K}$. The dimensions of the sample were $4.1 \mathrm{~mm} \times 3.7 \mathrm{~mm}$ with a thickness of $29 \mu \mathrm{m}$. Point contacts were placed at the surface, separated by $3.3 \mathrm{~mm}$. The sample was biased in series with a load resistor of $1 \mathrm{kohm}$ to an adjustable constant voltage source. The current was recorded from the voltage across the sample by broadband electronics. Proper biasing conditions in the post-breakdown regime were chosen to obtain one stable current filament. In order to reveal the spatial structure of the current flow through the sample a scanning optical microscope was applied [15]. The sample surface is scanned with a focused He-Ne-laser beam line by line and the photoconductiv response is recorded synchronously as a function of the irradiation position. Scanning is achieved by a 
mechanical deflection unit. Focusing of the irradiation is obtained by a lens of $30 \mathrm{~mm}$ focal length, mounted inside the optical cryostat and placed in front of the sample. A schematic drawing of the experimental setup is shown in Fig.1. Attenuators were used to vary the intensity of the laser beam which becomes monomode by passing a spatial filter. The deflection unit consists of a rotating drum carrying 16 separately adjustable plane mirrors. In order to scan different lines the drum can be tilted around a horizontal axis, lying in the front surface of the mirror in reflecting position. The beam splitter serves to place the center of curvature of the concave mirror on the beam deflecting plane mirror as well as in front of the center of the focusing lens. Thus the variable angle of reflection at the drum is transformed by the concave mirror into the necessary variable angle of incidence at the fixed focusing lens. Changing the angle of incidence causes a linear movement of the laser focus on the sample surface. The incident beam has a maximum power of $1 \mathrm{~mW}$ and a spot size of about $15 \mu \mathrm{m}$ at the sample. A computer is used to control the deflection unit, to record the transient photoconductivity by a waveform digitizer and to perform the reconstruction of the filament pattern.

In Fig.2a the photoconductive signal due to $\mathrm{He}-\mathrm{Ne}$ laser interband excitation is shown as a function of the position of the laser beam on the sample surface along line scans normal to the current flow. The measurements were carried out at a magnetic field strength of $B=0.1 \mathrm{~T}$. The orientation of $\vec{B}$ and the current flow $I$ with respect to the surface coordinates $(x, y)$ of the sam-

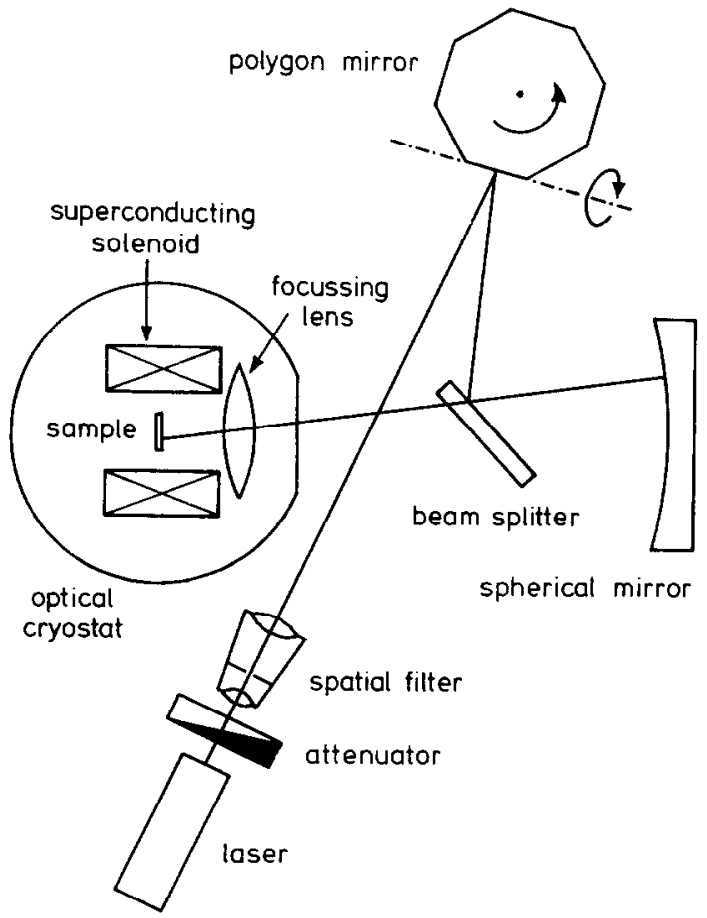

Fig. 1. Schematic drawing of the experimental arrangement for scanning the sample surface with a focused He-Ne-laser beam.

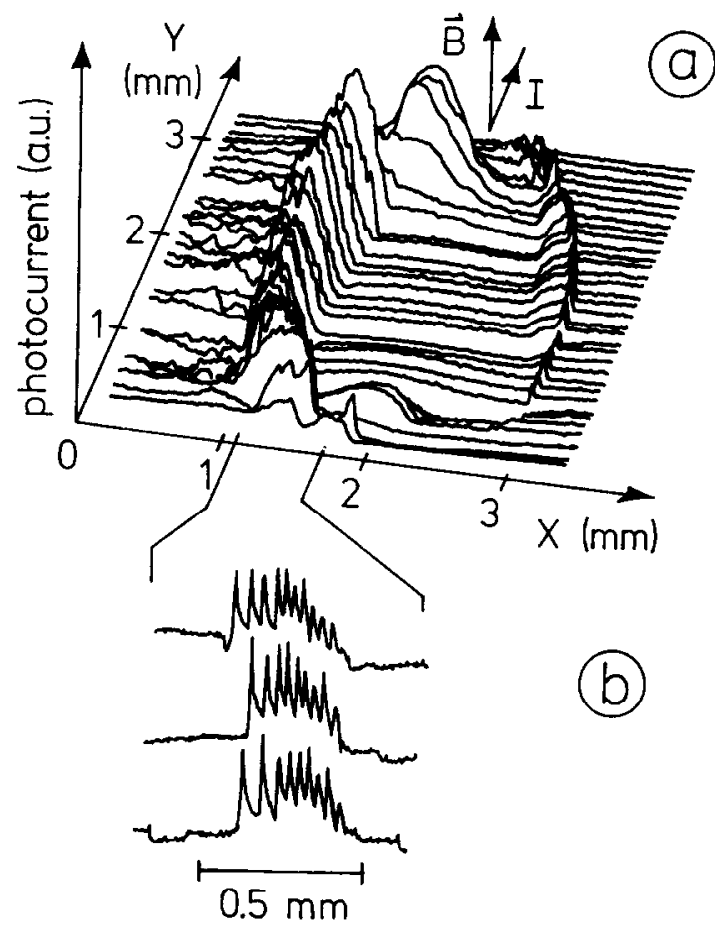

Fig. 2. (a) Reconstruction of a current filament in an n-GaAs epitaxial layer at a bias current of $0.9 \mathrm{~mA}$ and a magnetic field $B=0.1 \mathrm{~T}$. The signal heights are the photocurrent detected as function of the position of interband excitation on the sample surface. (b) Enlarged section of the left photosignal ridge for scanning three different lines demonstrating fluctuations.

ple are indicaled in the figure. The spatially resolved photocurrent shows two ridge-like structures, a strong ridge on the left side and a smaller one on the right side. In the following left and right is with respect to the orientation of $\vec{B}$ and $I$ in Fig. 2a. These ridges are due to photocarrier multiplication in the filament boundaries, whereas the asymmetry in heights and widths of these structures is caused by the magnetic field [15]. Thus the ridges indicate the lateral extent of the filament. Blowing up a ridge-like structure, as shown in Fig. $2 b$ for the large left ridge, reveals an oscillatory instability in the photocurrent when scanning this transition zone from the low-conducting region well outside the filament to the high-conducting region of the filament itself. Here current oscillations are induced in the otherwise stable current flow if the laser beam is acanned acroms the filament boundaries. Oscillations occur as long as the laser focus moves within a certain spatial interval approximately coinciding with the extent of the photosignal ridges. The same type of ocillations arise on both sides of the filament, however, the observed amplitudes and frequencies are smaller for the small ridge. Stationary focusing of the laser beam on a filament border causes oscillations, whereas when irradiating the sample well inside and outside the filament no current fluctuations were observed. The time dependence of the current is plotted in Fig.3 for different positions of the laser focus 


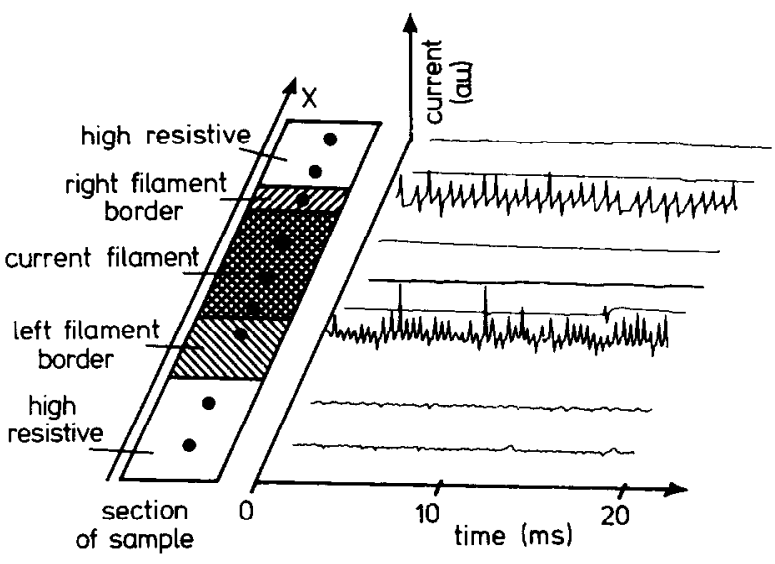

Fig. 3. Temporal behavior of the current fuctuations $\alpha$ curring for a fixed position of focused interband irradiation along a line scan across the current filament, obtained for a current of $0.22 \mathrm{~mA}$ and $B=1.75 \mathrm{~T}$. The positions of irradiation are marked by dark spots on a schematic section of sample.

in the filament geometry. The obeerved oscillations only set in above a critical irradiation intensity. The amplitude of these current fluctuations is practically unaltered by the irradiation intensity for the left border, whereas it increases slightly with rising intensity for the right border. The fundamental frequencies of the oscillations are different for both filament boundaries, faster oscillations occur when irradiating the left boundary showing the stronger photoconductivity ridge. The spatial extent on the $x$-axis, where current fluctuation may be induced by interband excitation, grows with increasing intensity on both sides of the filament, whereas it grows on the left side and shrinks on the right side when the magnetic field strength is increased.

The observed phenomena may be understood qualitatively in terms of the nonlinear generation-recombination kinetics due to impact ionization of shallow impurities by taking into account the formation of a Hall field across the current filament [16]. Once a stable filament is formed in a transverse magnetic field, charge neutrality is lifted in the filament boundaries by accumulation and depletion of electrons on the right and left side of the filament, respectively, yielding the source and drain of the Hall field inside the sample. The combined effect of the Lorentz force, the Hall field and the wellknown $1 / B$ dependence of the electron diffusion length on the magnetic field strength narrows the right boundary and widens the left boundary of the filament compared to the zero magnetic field case $[15,17]$. In the transition zones between filament and high resistive outside regions of the sample a large population of excited donor states is established. This situation in concentration profiles along a line scan across the current filament is sketched schematically in Fig.4 together with a simplified energy level diagram indicating the relevant electron generation and recombination processes. Interband irradiation will generate additional free electrons. This

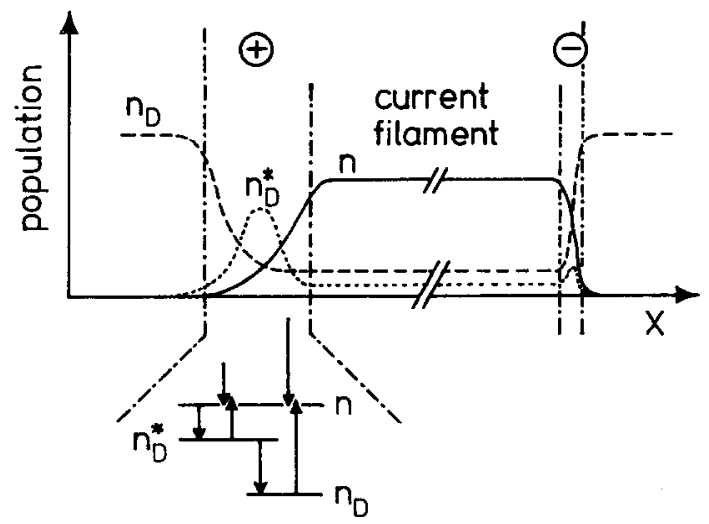

Fig. 4. Schematic concentration profile of free carrier $n$, excited donors $n_{D}^{*}$ and donors in ground state $n_{D}$ along a line scan across a current filament. The insert shows the most important impact ionization generation and relaxation processes occurring in the transition zones marked with $\oplus$ and $\theta$ according to the formation of the Hall field in a transversal magnetic field.

local increase in the free carrier concentration in conjunction with an increased population of excited shallow donors in the transition zones will trigger an avalanche in the generation of free elextrons by the impact ionization process. From this point of view the number of free carriers produced by interband transitions is multiplied by the impact ionization of shallow impurities. The resultant situation is not a steady state, so reestablishing of the former situation is recovered through relaxation of the electrons into the donor states and into the valence band. By cyclic repetition of this process, described above, an autonomous current fluctuation is obtained for irradiating the transition zones. For the right narrow transition zone the basin of the population of excited donor states is smaller, so multiplication of interband generated electrons will be less effective. As a consequence the peak response in the photocurrent and the width where current oecillations may be induced are smaller for the right transition zone in comparison to the left one. The characteristic time of retriggering the impact ionization avalanche is given by the relaxation time to a concentration profile capable of destabilizing the generation-recombination kinetics. For the narrower right transition zone this time will be longer, due to a greater accumulation of free carriers generated by interband irradiation in combination with a smaller basin of ionised impurities relaxation occurs into. So different frequency characteristics are obtained for each of the borders of the current filament. The magnetic field adjusts the width of the transition zones and the population of excited donor states at the filament boundary and hence the peak response and the fundamental frequencies in the current oacillations. So the frequencies of the current fluctuations become a function of the magnetic field. Impact ionization of excited states of shallow impurities will introduce a small high-frequency current oscillation whereas impact ionization from the ground state of shallow impurities will lead to pronounced cur- 
rent fluctuations which can be regarded as an oecillation in the width of the current. Breathing filaments due to oscillations in space charge zones were theoretically anticipated by Schöll [18].

In conclusion, the spatial pattern of current filaments in $\mathrm{n}-\mathrm{GaAs}$ at low temperatures were reconstructed in a magnetic field by an optical method. A scanning optical microscope was developed which is based on interband excitation induced free carrier multiplication. In a stable filamentary current flow, fluctuations were observed when irradiating the filament boundaries whereas no fluctuation could be found for exciting the filament itself or the high resistive regions outside. The fundamental frequencies, the strength of response and the spatial width, where current fluctuations may be initiated, are different for opposite edges of the filament characterized by electron depletion and accumulation yielding source and drain of a Hall field. This difference in the generation-recombination kinetic for different regions of the sample is introduced by an external magnetic field and may be the cause of previously observed spontaneous multimodal oscillations like quasiperiodic, frequency-locked and chaotic current fluctuations.

Acknowledgements - We thank E. Bauser, Max-PlanckInstitut für Festkörperforschung, Stuttgart, for provision of the samples and E. Schöll for valuable discussions. Financial support by the Deutsche Forschungsgemeinschaft is gratefully acknowledged.

\section{REFERENCES}

1. R. Obermaier, W. Böhm, W. Prettl, and P. Dirnhofer: Phys. Lett. A 105, 149 (1984); M. Weispfenning, I. Hoeser, W. Böhm, W. Prettl, and E. Schöll: Phys. Rev. Lett. 55, 754 (1985).

2. E. Schöll: Z. Phys. B 52, 321 (1983).

3. B. Rōhricht, R.P. Huebener, J. Parisi, and M. Weise: Phys. Rev. Lett. 61, 2600 (1988).

4. S.W. Teitsworth, R.M. Westervelt, and E.E. Haller: Phys. Rev. Lett. 51, 825 (1983).

5. G.A. Held, C. Jeffries, and E.E. Haller: Phys. Rev. Lett. 52, 1037 (1984).

6. A. Brandl, T. Geisel, and W. Prettl: Europhys. Lett. 3, 401 (1987).

7. J. Peinke, J. Parisi, B. Röhricht, K.M. Mayer, U. Rau, and R.P. Huebener: Solid-State Electr. 31, 817 (1988), and references therein.

8. J. Spangler, A. Brandl, and W. Prettl: Appl. Phys. A 48, 143 (1989).

9. U. Frank, A. Brandl, and W. Prettl: Solid State Commun. 69, 891 (1989).
10. K. Aoki, and K. Yamamoto: Appl. Phys. A 48, 111 (1989).

11. A.M. Barnett and A.G. Milnes: J. Appl. Phys. 37, 4215 (1966).

12. L.P. Zverev, G.M. Min'kov, and N.K. Sumin: Sov. Phys. Semicond. 8, 1457 (1975).

13. H. Baumann, R. Symanczyk, C. Radehaus, H.-G. Purwins, and D. Jäger: Phys. Lett. A 123, 421 (1987).

14. K.M. Mayer, R. Gross, J. Parisi, J. Peinke, and R.P. Huebener: Solid State Commun. 63, 55 (1987); K.M. Mayer, J. Parisi, J. Peinke, and R.P. Huebener: Physica $D$ 32, 306 (1988).

15. A. Brandl, M. Völcker, and W. Prettl: Appl. Phys. Lett., 55, 238 (1989).

16. A. Brandl, W. Krōninger, W. Prettl, and G. Ober mair; to be published.

17. H. Bruhns, K. Hübner, A. Neidig, L. Schenk, and W. Schneider: Appl. Phys. 10, 33 (1976).

18. E. Schöll: Physica Scripta, to be published. 